\title{
Small World Patterns in Food Webs
}

\author{
Jose M. Montoya ${ }^{1,2}$ and Ricard V. Solé ${ }^{1,3}$ \\ 1 Complex Systems Research Group, FEN \\ Universitat Politècnica de Catalunya, Campus Nord B4, 08034 Barcelona, Spain \\ ${ }^{2}$ Intercampus Department of Ecology, University of Alcalá \\ 28871 Alcalá de Henares, Madrid, Spain \\ ${ }^{3}$ Santa Fe Institute, 1399 Hyde Park Road, New Mexico 87501, USA
}

\begin{abstract}
The analysis of some species-rich, well-defined food webs shows that they display the so called Small World behavior shared by a number of disparate complex systems. The three systems analysed (Ythan estuary web, Silwood web and the Little Rock lake web) have different levels of taxonomic resolution, but all of them involve high clustering and short path lengths between species. Additionally, the distribution of connections $P(k)$ is skewed in all the webs analysed and shows a power-law behavior $P(k) \propto k^{-\gamma}$ in two cases (with $\gamma \approx 1$ ). These features suggest that communities might be self-organized in such a way that high homeostasis to perturbations (with short transient times to recovery) would be at work. The consequences for ecological theory are outlined.
\end{abstract}

\section{INTRODUCTION}

The understanding of food web patterns in complex ecologies is a central issue in ecology. As Stuart Pimm claims, food webs are the road-maps through Darwin's entangled bank (Pimm et al, 1991). Beyond the specific features of currently described food webs (such as the concrete species composition) a number of regularities have been observed suggestive of fundamental laws of ecosystem organization (see for example Warren, 1994; Polis and Strong, 1996 and references therein).

One of the most successful approaches to real food webs deals with the graph patterns associated with the set of links between species. Specifically, a food web can be described in terms of a graph $G(V, E)$ consisting of a finite set $V$ of vertices (nodes, species) and a finite set $E$ of edges such that each edge $e$ is associated with a pair of vertices $v$ and $w$. In spite that ecological graphs are directed (i. e. links go from one species to another and the reverse connection will be typically different in strength and sign) most theoretical studies deal with non-directed graphs. In other words, two species appear connected if they share a common edge, irrespective of its particular properties. This approach has been rather successful, since many reported regularities form field data are fully recovered from non-directed graph arguments of different nature (Cohen et al., 1990; Pimm et al., 1991; Williams and Martinez, 2000; see also Albert et al. 2000). This observation is probably a consequence of fundamental laws constraining the basic topological arrangements of ecological networks that go beyond the specific rules of dynamical interaction (Brown, 1994; Solé and Bascompte, 2001).

Recently, new theoretical approaches to graph complexity in nature have emerged. Two main results of these studies are: (a) the widespread presence of the so called small world (SW) pattern of some economic, technologic and biological networks (Watts and Strogatz, 1998; Watts, 1999; Adamic, 1999) and (b) the pres- ence of scale-free (SF) distributions $P(k)$ of connections (Barabási and Albert, 1999; Jeong et al., 2000; Wagner and Fell, 2000). Specifically, in some cases the number of nodes with $k$ links follows a power law distribution $P(k) \sim k^{-\gamma}$ where most units are weakly connected and a few are highly linked to other nodes. This is however a non-universal feature: the graph of the neural network of $C$. elegans, for example, displays an exponential distribution of connections (Amaral et al., 2000).

The SW pattern can be detected from the analysis of two basic statistical properties: the so called clustering coefficient $C_{v}$ and the path length $L$. Let us consider the set of links $\xi_{i j}(i, j,=1, \ldots, S)$ where $S$ is the number of species and let's assume that $\xi_{i j}=1$ if a link exist and zero otherwise. Species will be labeled as $s_{i}(i=1, \ldots, S)$. If a food web is considered, an additional parameter is the community matrix connectance $C$. Let us consider a given species (the i-th one) and the set of nearest neighbors $\Gamma_{i}=\left\{s_{i} \mid \xi_{i j}=1\right\}$. Here we can calculate the clustering coefficient for this species as the number of connections between the species belonging to $\Gamma_{i}$. By defining

$$
\mathcal{L}_{i}=\sum_{j=1}^{S} \xi_{i j}\left[\sum_{k \in \Gamma_{i}} \xi_{j k}\right]
$$

and thus $c_{v}(i)=\mathcal{L}_{i} /(C S(C S-1) / 2)$ so that the clustering coefficient is the average over all species:

$$
C_{v}=\frac{1}{S} \sum_{i=1}^{S} c_{v}(i)
$$

and measures the average fraction of pairs of neighbors of a node that are also neighbors of each other.

The second measure is easily defined. Given two arbitrary species $s_{i}$ and $s_{j}$, let $L_{\min }(i, j)$ be the minimum path length connecting these two species. The average path length $L$ will be:

$$
L=\frac{2}{S(S-1)} \sum_{i=1}^{S} \sum_{j=1}^{S} L_{m i n}(i, j)
$$


Small World graphs are highly clustered but the minimum path length between any two randomly chosen nodes in the graph is short. By comparison, random graphs (where nodes are randomly connected with some probability) are not clustered and have short $L$ (Watts, 1999). At the other extreme, regular lattices are typically clustered and have long distances. It has been shown, however, that a regular lattice can be transformed in to a SW if a small fraction of nodes are rewired to randomly chosen nodes. Thus a small degree of disorder makes the lattice to have short paths (as in the random case) but is still mostly regular (Watts and Strogatz, 1998).

In a graph with an average of $\langle k\rangle$ links per node, it can be shown for random graphs that $C_{v}^{\text {rand }} \approx<k>/ N$ and $L^{\text {rand }}$ will be short. For large networks, a SW is present if $L^{\text {rand }}$ is larger, but close to $L$ and $C_{v} \gg C_{v}^{r a n d}$. When networks composed by a small number of units are analysed, the second condition is often replaced by $C_{v}>C_{v}^{r a n d}$. This is the case for the SW pattern in the neural network of the nematode worm $C$. elegans (ce) which has 282 neurons and $C_{v}^{c e}=0.28$ and $L^{c e}=2.65$, to be compared with the corresponding random graphs: $C_{v}^{r a n d}=0.05$ and $L^{\text {rand }}=2.25$. For the metabolic network of E. coli, Wagner and Fell obtained similar results, but with a much larger difference in the clustering.

The consequences of the SW and SF paterns are far from trivial and can be of great importance in recognizing evolutionary paths, the origins of homeostatic stability and the sensibility to perturbations in biological networks. Watts and Strogatz discuss some of these ideas in their seminal work, suggesting that a SW architecture would play a relevant role in enhancing synchronization in the visual cortex. For metabolic networks, Wagner and Fell suggest that it might play a relevant role in allowing metabolism to react rapidly to perturbations thus displaying a very high homeostasis. What about ecological networks? Do food webs display this type of topological properties?

In this paper we explore this question by analysing the statistical properties of a set of large $(S>100)$ ecological networks where a fine taxonomic resolution is available. For these webs, the SW property is shown to be present and a skewed distribution of links is also shown to be involved in most cases. The presence of these properties will be shown to have deep implications for theoretical and applied issues at different levels.

\section{ECOLOGICAL SMALL WORLDS}

It is interesting to see that in fact most static graph models of food webs involve features ranging from purely random nets (constant connectivity networks, see Martinez, 1991a) to purely hierarchical models (the cascade model, see Cohen et al., 1990). Under the second class, a trophic species can only prey on a trophic species of lesser rank leading to a hierarchical structure sharing some fea- tures with some standard graph patterns such as Cayley trees (Watts, 1999). For the constant connectance model (Martinez, 1991a) no such ranking is introduced and a trophic species preys on any other trophic species (including itself). However, ecological nets display both types of properties and some studies suggest that they also display clustering.

Evidence for clustering in food webs has been compiled in recent years (see Solow and Beet, 1998). A number of authors have shown that food webs are typically non-random but the effects such topological properties on stability are far from clear (see Solow et al., 1999, for a recent discussion).
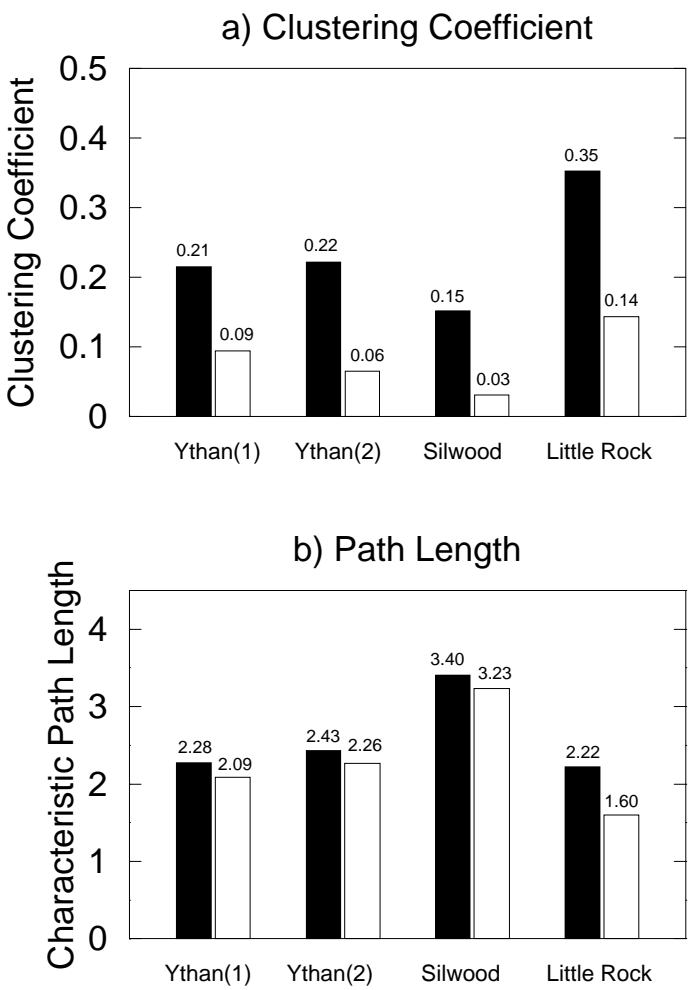

FIG. 1. (a) clustering coefficient for the four analysed food webs (see text). Here the dark bars are real webs and white bars correspond to randomly generated webs with the same average number of links per species (averaged over 200 generated samples). We can see that in all cases the clustering is clearly larger than random; (b) Characteristic path length $L$ with random values. Except for Little Rock, the difference between the random and real case is very small. For the two Ythan webs, the improvement in resolution slightly modified the observed relations towards a better defined SW pattern.

The possible importance for clustering on the stability of ecological networks was already recognized from early statistical approaches (May, 1974; Pimm, 1982). Given the potential implications of the SW phenomenon for network homeostasis, the presence of such properties in real food webs would be of great interest in this discussion. We have found that, in fact, rich-species food webs with a 
good taxonomic resolution display the properties of small world behavior. In figure (1) we show the obtained results for the four large food webs analyzed, which are known in the literature because they are the most detailed large community food webs available. These are:

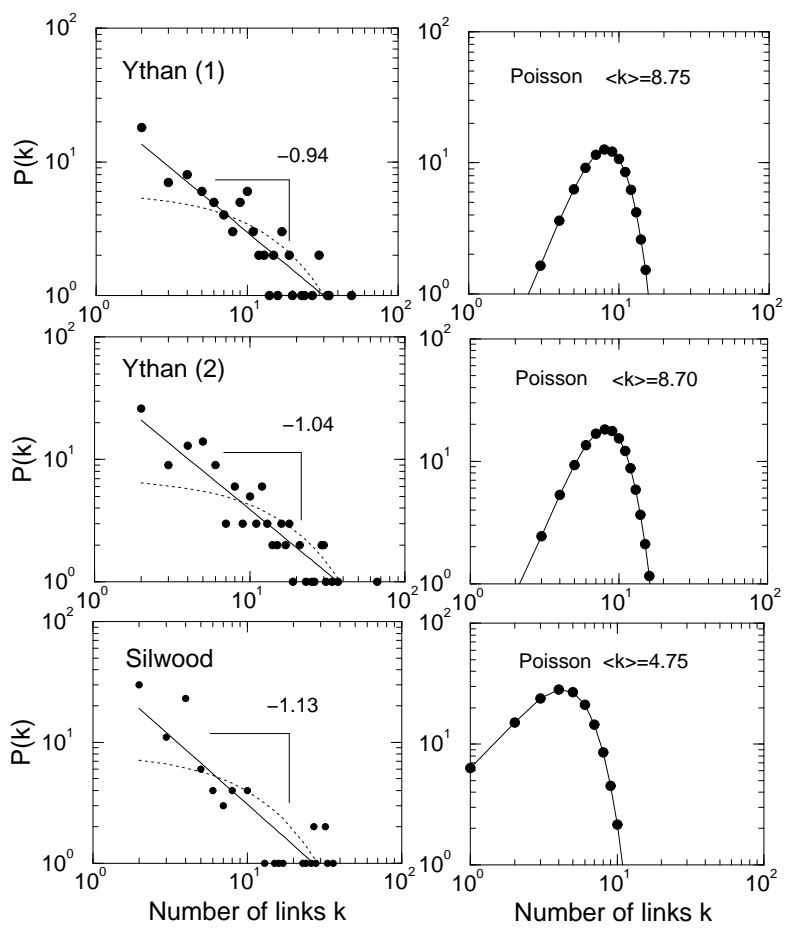

FIG. 2. Frequency distributions of links $P(k)$ for the analysed webs. Left column: actual data, which fit quite well a power law for the Ythan and Silwood cases (the exponential fit is also shown as a dotted line). The improvement in taxonomic resolution for Ythan (2) only modifies the exponent slightly. Right column: Expected distributions from the random graph approximation, predicting Poissonian behavior. None of the analysed food webs can be approached by the random case.

1. Ythan estuary: this web is from a freshwatermarine interface. The number of species reported from two different studies are $S=93$ (Hall and Raffaelli, 1991) and $S=134$ (Huxham et al., 1996). These studies differ in the taxonomic detail incorporated and we use them in order to check the sensitivity in relation with the measurement of SW and scaling properties. This is the largest documented web in the UK and the average number of links per species are $\langle k\rangle=8.75$ and $\langle k\rangle=8.70$, respectively. The second web was expanded by adding 42 metazoan parasite species but their effect on $\langle k\rangle$ was weak. For this web most $(88 \%)$ nodes correspond to real species. Some nodes involve lower resolution at different levels (Nematodes, Acarina or Brown algae, for example, are lumped together).

2. Silwood park web (Memmott et al., 2000): this is a sub-web obtained from a field site of 97 hectares in size. This is a very detailed community centred on the Scotch broom Cytisus scoparius, involving $S=154$ species: one plant, 19 herbivores, 5 omnivores, 66 parasitoids, 60 predators and three pathogens. The average connectivity is $<k>=4.75$. All nodes of this (sub-) network are species (except node 122: immature spiders).

3. Little Rock lake (Martinez, 1991): a web from a freshwater habitat, it includes $S=182$ species. The Little Rock lake is a small lake with an area of 18 hectares. Here $\langle k\rangle=26.05$. For this web, only $31 \%$ of nodes are associated to species. Most are genera-level nodes (63\%) and the rest correspond to higher taxa (Bivalvia, Hirudinea, etc).

We compared the observed values of $C_{v}$ and $L$ with the expected ones from the randomized webs with the same number of (total) links. As expected from a SW pattern, we see that all have very similar (and very short) distances $L$ and a clustering coeficient from $C_{v}^{Y \text { th1 }} / C_{v}^{\text {rand }}=$ 2.33 to $C_{v}^{\text {Silw }} / C_{v}^{\text {rand }}=5.0$ (numerically close to the $C$. elegans data) i. e. clearly larger than expected from random webs. For the average path length, we found that the values are very close except for the Little Rock case, were notable differences are present.

An additional and surprising feature of the observed distributions of connections $P(k)$ is that they show strongly non-Poissonian behavior (wich would be expected from random wiring). The classic result by Erdös and Rényi (ER) on random graphs (see Bollobás, 1985) with $S$ nodes shows that the probability that a vertex has $k$ edges follows a Poisson distribution $P(k)=$ $e^{-<k>} \lambda^{k} / k$ !. Here

$$
<k>=\left(\begin{array}{c}
S-1 \\
k
\end{array}\right) P_{r}^{k}\left(1-P_{r}\right)^{S-k-1}
$$

where $P_{r}$ is the probability that two nodes are connected. The observed distributions for Ythan webs and the Silwood example are shown in figure 2 (left column). In both webs, the distribution was found to be strongly skewed with a good agreement with a power law fit. The observed values for a regression of the log-transformed data are:

$$
\begin{array}{ll}
\gamma^{Y t h 1}=0.94 \pm 0.06 & \left(r^{2}=0.79, p<0.01\right) \\
\gamma^{Y t h 2}=1.04 \pm 0.05 & \left(r^{2}=0.83, p<0.01\right) \\
\gamma^{\text {Silw }}=1.13 \pm 0.06 & \left(r^{2}=0.79, p<0.01\right)
\end{array}
$$

For comparison, the expected connectivity distributions from a random, Poissonian net are also shown in Figure 2 (right column). Here the average number of links per species in each web was used. 
The Little Rock web (figure 3) shows a fluctuating distribution of connections with a very high variance, but no obvious recognizable standard shape, although it is also skewed, with a prominent peak of 21 species (involving a coarse-grained cluster of cyanobacteria and green algae predated by pelagic cladocera, copepods and rotifers) with $k=26$ links. These anomalies are likely to due to the taxonomic resolution used (mostly at genera or higher levels).

The exponent derived from our study is rather different from the estimations obtained for Internet or the metabolic graphs suggesting that, in spite that the SW pattern together with the scaling law might be common to many different types of networks, the ecological webs belong to some different class of complex phenomena (Solé et al., 1996) to be defined in future theoretical studies.

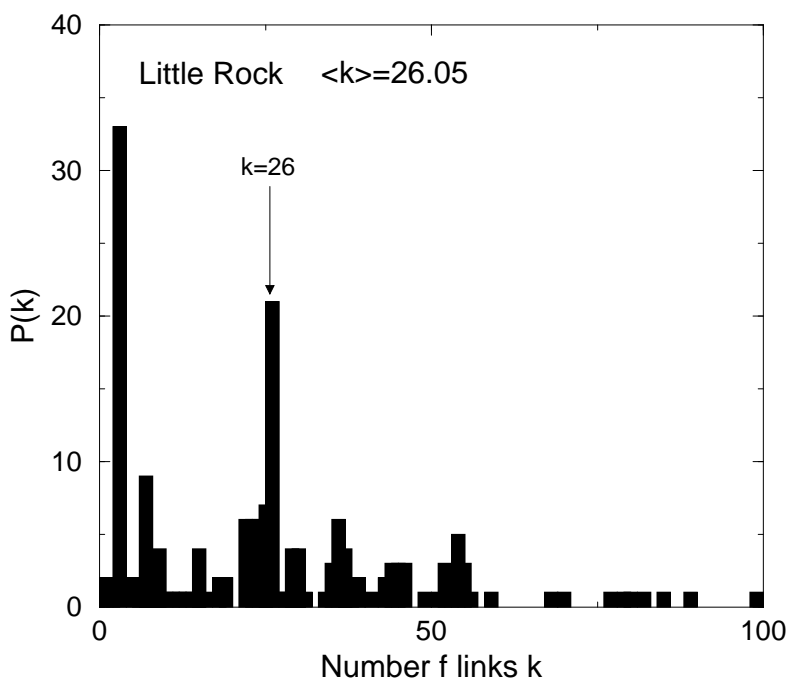

FIG. 3. Little Rock lake link distribution. Here $P(k)$ is clearly skewed but strong fluctuations are observable as scattered peaks, in particular a large peak at $k=26$ involving a large cluster of interacting species (see text). Other peaks are indicated, revealing strong deviations from a monotonous power-law tail.

\section{DISCUSSION}

In this paper we have presented evidence for SW patterns in ecological networks. The aim of our study was to see to what extent such evidence was strong enough by analysing a small number of large, taxonomically welldefined food webs from the ecological literature. The webs analysed have different origins but all of them share the SW pattern in their graphs and are clearly away from their random counterparts. As far as we know, this is the first reported evidence for such property in ecology. It reveals, by means of a new measure, the existence of clustering in food webs associated to short path lengths and opens a number of questions not previously formulated in previous theoretical studies.

Although some food web analyses found evidence for the existence of compartments (Solow and Beet 1998), such species clusters were established in terms of the trophic similarity between pairs of species (see also Pimm and Lawton 1980, for a similar measure). In contrast, the aim of the clustering coefficient as a measure of compartmentalization is to find out to what extent groups of species are more connected internally than they are with another species or groups of species. In other words, this new measure go beyond by looking not only for resourceuse-guilds (see Wilson, 1999) but also for sub-webs with higher internal connectance than the rest of the community.

The use of the clustering coefficient is supported by the fact that groups of species with diets of similar species do not occur, especially in species-rich systems, such as those analysed in this article (Winemiller 1990; Polis and Strong 1996). In this respect, this measure of compartmentalization minimize the importance of discrete and homogeneous trophic-level dynamics in complex food webs, an issue widely considered as an artifact by many ecologists (see Polis and Strong, 1996, for a review). The clustering coefficient does not consider the trophic position of the species in the food web, focusing only in the presence or absence of the interactions.

Two of the webs analysed correspond to the same system: the Ythan estuary food web. These two webs involve different taxonomic resolution but the exponents and indices remained basically the same for the two cases. This agreement suggests that the SW property and the scaling law are rather robust and that no completely detailed description of the species-level graph needs to be defined in order to detect these properties. For the best defined web (Silwood), where all nodes belong to species, we obtain the best evidence of SW, suggesting that lessdetailed webs introduce a coarse-grained resolution that might hidden a higher degree of clustering (this seems to be confirmed by the Ythan example). The worst result is given by Little Rock lake, where the resolution is mainly at the genus level and strong deviations from the power law are observable.

The SW phenomenon appears to be associated with highly non-random, fat-tailed distributions of links. The plots of $P(k)$ show that all food webs (and others not explored here due to their lower taxonomic resolution) involve strong deviations from the Poissonian behavior and confirm the presence of clustering in ecological interactions. These properties reveal that some new features of ecological graphs have to be considered by future models in order to explain their origin and meaning.

In our system, the presence of such a web pattern might have important consequences for stability and resilience. Species removal (Brown and Heske, 1990; Brown, 1994) or the introduction of sharp shocks in population abundances due to transient perturbations might be strongly damped through the rapid transfer of information characteristic of these webs (Watts and 
Strogatz, 1998). In this sense, classic studies on ecosystem resilience and resistance might benefit from considering these topological constraints. Most studies suggest that the characteristic time of recovery from short, sharp shocks (as it would be the case for a pulse of nutrients) depends on the lenght of the food chain. Some enlightening theoretical studies based on the analysis of small-species models show this trend (Pimm and Lawton, 1977) where some simple topological arrangements between species are predefined. But perhaps some other regularities will emerge from our results when rich-species models are taken into account.

A different question emerging from our study is how such food webs are constructed through community assembly rules (Drake, 1990a, 1990b). Since membership in a community is usually influenced by interactions among species, the dynamical process leading to a SW pattern of connections might eventually allow to understand how our reported results are explained. A possible dynamical process underlying the presence of scaling in food webs is preferential attachement (Barabási and Albert, 1999). This process of assembly consists in the addittion of new nodes which are preferentially linked to already highly-connected nodes. This process has been argued as the explanatory mechanism of the organization of different complex networks, including technological ones or the large set of metabolic networks analysed by Jeong and collaborators (2000). But for ecological networks (represented as food webs) is far from trivial to decide if preferential attachement is an appropriate, general assembly rule. The ubiquity of assembly rules has been recently discussed (Weiher and Keddy 1999) almost each one corresponding to each of the different ecological communities studied.

Even if a community is assembled by random addition of species, interactions can lead to non-random webs. If network homeostasis is a global constraint to species richness, then a SW pattern might be an 'attractor' in community assembly (Weiher and Keddy, 1999). Future models will require to incorporate weighted, directed links and the scaling behavior of these graphs can have some important effects in extinction dynamics (Solé and Montoya, in preparation) and might lead to some unexpected results. The SW topology might be related to underlying community level constraints and might help understanding how biodiversity emerges and persists.

\section{Acknowledgments}

The authors thank Javier Gamarra and David Alonso for help in the analysis of some of the food webs and useful "night crew" discussions on theoretical ecology. Special thanks to Ramon Ferrer for his help with the graph analysis. We also thank Miguel Angel Rodriguez for useful talks and a careful reading of the manuscript. This work has been supported by a grant CICYT PB97-0693 and The Santa Fe Institute (RVS).
1. Adamic L. A. (1999) The small world web. Proceedings of the ECDL'99 Conference, pp.443-452 (Springer, Berlin).

2. Albert, R., Jeong, H. and Barabasi, A-L (2000) Error and attack tolerance of complex networks. Nature 406, 378-382. Jeong, H., Tombor, B., Albert, R., Oltvai, Z.N. and Barabasi, A-L. (2000) The large scale organization of metabolic networks. Nature 407, 651-654.

3. Amaral, L. A. N., Scala, A., Barthélemy, M. and Stanley, H. E. (2000) Classes of behavior of smallworld networks. Proc. Nat. Acad. Sc. USA 97, 11149-11152.

4. Barabási, L. A. and Albert, R. (1999) Emergence of Scaling in Random Networks, Science, 286, 509512

5. Bollobás, B. (1985) Random Graphs. (Academic Press, London)

6. Brown, J. H. and Heske, E. J. (1990) Control of a desert-grassland transition by a keystone rodent guild. Science, 250, 1705-1707

7. Brown, J. H. (1994) Complex ecological systems, in Complexity: Metaphors, Models and Reality Cowan, G., Pines, D., and Meltzer, D., eds., pp. 419-449, Addison Wesley.

8. Cohen, J. E., Briand, F. and Newman, C. M. (1990) Community Food Webs: Data and Theory. Biomathematics, vol. 20 (Springer, Berlin)

9. Drake, J. A. (1990a) The mechanics of community assembly rules. J. Theor. Biol., 147, 213-233.

10. Drake, J. A. (1990b) Communities as assembled structures: do rules govern pattern?. Trends Ecol. Evol., 5, 159-163.

11. Hall, S. J. and Raffaelli, D. (1991) Food web patterns: lessons from a species-rich web. J. Anim. Ecol. , 60, 823-842

12. Hanski, I. (1999) Metapopulation Ecology, Oxford U. Press.

13. Huxham, M., Beaney, S. and Raffaelli, D. (1996) Do parasites reduce the chances of triangulation in real food webs? OIKOS 76, 284-300

14. Martinez, N. D. (1991a) Constant connectance in community food webs. Am. Nat. 139, 1208-121.

15. Martinez, N. D. (1991b) Artifacts or attributes? Effects of resolution on the Little Rock lake food web. Ecol. Monographs 61, 367-392. 
16. May, R.M. (1974) Stability and complexity in model ecosystems. Princeton U. Press.

17. Memmott, J., Martinez, N. D. and Cohen, J. E. (2000) Predators, parasitoids and pathogens: species richness, trophic generality and body sizes in a natural food web. J. Anim. Ecol. 69, 1-15

18. Pimm, S. L. and Lawton, J. H. (1977) The number of trophic levels in ecological communities. Nature (Lond.) 268, 329-331

19. Pimm, S. L. and Lawton, J. H. (1980) Are food webs divided into compartments? J. Anim. Ecol. , 49, 879-898

20. Pimm, S. L. (1982) Ecological Food Webs, Chapman and Hall.

21. Pimm, S. L., Lawton, J. H. and Cohen, J. E. (1991) Food web patterns and their consequences. Nature (Lond.) 350, 669-674.

22. Pimm, S. L. (1991) The Balance of Nature. Chicago Press.

23. Polis, G.A. and Strong, D.R. (1996) Food web complexity and community dynamics. American Naturalist 147, 813-846.

24. Solé, R. V. et al. (1996) Phase Transitions and Complex Systems, Complexity 1 (4), 13-18

25. Solé, R. V. and Bascompte, J. (2001) Complexity and Self-organization in Evolutionary Ecology, Monographs in Population Biology. (Princeton U. Press) (to appear)
26. Solow, A. R. and Beet, A. R. (1998) On lumping species in food webs. Ecology 79, 2013-2018

27. Solow, A. R., Costello, C. and Beet, A. R. (1999) On an early result on stability and complexity. $\mathrm{Am}$. Nat. 154, 587-588

28. Wagner, A. and Fell, D. (2000) The small world inside large metabolic networks, Santa Fe Institute Working Paper 00-07-041.

29. Warren, P. H. (1994) Making connections in food webs. Trends Ecol. Evol. 4, 136-140

30. Watts, D. J. (1999) Small Worlds. Princeton U. Press

31. Watts, D. J. and Strogatz, S. H. (1998) Collective Dynamics in 'small-world' networks, Nature (Lond.) 393, 440-442

32. Weiher, E. and Keddy, P., eds. (1999) Ecological Assembly Rules. Cambridge U. Press

33. Winemiller, K.O. (1990) Spatial and temporal variation in tropical fish trophic networks. Ecol. Monog. bf60, 331-367.

34. Williams, R. J. and Martinez, N. D. (2000) Simple rules yield complex food webs. Nature (Lond.) 404, 180-183

35. Wilson, J.W. (1999) Guilds, functional types and ecological groups. Oikos 86, 507-522. 